\title{
The Energy Content of Extensive Air Showers in the Radio Frequency Range of 30-80 MHz
}

\author{
Christian Glaser ${ }^{* a}$ for the Pierre Auger Collaboration ${ }^{b}$ \\ ${ }^{a}$ RWTH Aachen University, Aachen, Germany \\ ${ }^{b}$ Observatorio Pierre Auger, Av. San Martín Norte 304, 5613 Malargüe, Argentina \\ E-mail: auger_spokespersons@fnal.gov \\ Full author list: http://www.auger.org/archive/authors_2015_06.html
}

\begin{abstract}
At the Auger Engineering Radio Array (AERA) of the Pierre Auger Observatory, we have developed a new method to measure the total amount of energy that is transferred from the primary cosmic ray into radio emission. We find that this radiation energy is an estimator of the cosmic ray energy. It scales quadratically with the cosmic ray energy, as expected for coherent emission. We measure $15.8 \mathrm{MeV}$ of radiation energy for a $1 \mathrm{EeV}$ air shower arriving perpendicular to the geomagnetic field at the Auger site, in the frequency band of the detector from 30 to $80 \mathrm{MHz}$. These observations are compared to the data of the surface detector of the Observatory, which provide well-calibrated energies and arrival directions of the cosmic rays. We find energy resolutions of the radio reconstruction of $22 \%$ for the complete data set, and $17 \%$ for a high-quality subset containing only events with at least five stations with signal.
\end{abstract}

The 34th International Cosmic Ray Conference,

30 July - 6 August, 2015

The Hague, The Netherlands

${ }^{*}$ Speaker. 


\section{Introduction}

The detection of ultra-high energy cosmic rays via short radio pulses that are emitted during the air shower development has become an active field of research in recent years [1, 2, 3, 4, 5, 6, 7]. One advantage of this technique is a detection of cosmic rays that is sensitive to the particle type at close to a $100 \%$ duty cycle. The potential for a large scale radio detector is currently being explored in several experiments.

Much progress has been achieved in the understanding of the radio emission process. The dominant process is the geomagnetic emission that is due to the deflection of charged shower particles in the Earth's magnetic field which is polarized in the direction of the Lorentz force that acts on the shower particles $[1,3,8]$. The second component is polarized radially with respect to the axis of the air shower and results from the negative charge excess in the shower front $[9,10,11,12]$. Its relative strength is on average 14\% at the Auger site for an air shower arriving perpendicular to the geomagnetic field [13]. The constructive and destructive interference of the two emission processes lead to a radial asymmetry of the lateral signal distribution function (LDF) $[14,15,16]$ that can be modeled with a two-dimensional LDF [17, 18].

The Auger Engineering Radio Array (AERA) [19] at the Pierre Auger Observatory [20] is the world's largest cosmic ray radio detector. In its latest stage of expansion, it consists of 153 radio detector stations that cover an area of approximately $17 \mathrm{~km}^{2}$. AERA can take advantage of the fluorescence and surface detectors (SD) of the Pierre Auger Observatory that provide wellcalibrated energies and arrival directions of the cosmic rays. The radio detector (RD) stations of AERA are located in an area of denser detector spacing of the SD array. This region, with SD station spacing of $0.75 \mathrm{~km}$, allows the detection of cosmic ray energies down to about $0.1 \mathrm{EeV}$.

\section{Data selection and event reconstruction}

In this work we are using RD and SD data recorded between April 2011 and March 2013 when AERA was operating in its first phase. In this phase, AERA consisted of 24 antenna stations with a spacing of $144 \mathrm{~m}$. The stations are equipped with logarithmic-periodic dipole antennas and measure over a frequency range of 30 to $80 \mathrm{MHz}$ [21]. We use only events that have been measured by the radio and surface detectors in coincidence.

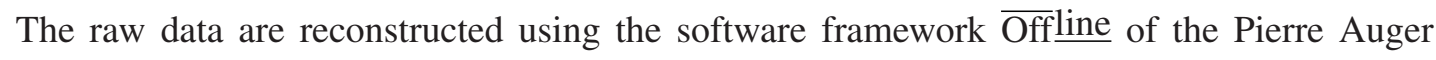
Collaboration $[22,23]$. We correct for the influence of the analog signal chain using the absolute calibration of the AERA station and reconstruct a three-dimensional electric field by using the direction of the shower and applying the simulated antenna response [21]. An example of a reconstructed electric field trace $\vec{E}(t)$ is shown in Fig. 1 .

We determine the energy density $u$ of the incoming electromagnetic radio pulse at each radio station by calculating the time integral over the absolute value of the Poynting vector. We add up the square of the magnitude of the electric field trace in a time-window of $200 \mathrm{~ns}\left(\left[t_{1}, t_{2}\right]\right)$ around the pulse maximum. The pulse maximum has been determined from the Hilbert envelope of the trace. We subtract the contribution of background noise (determined in the noise window $\left[t_{3}, t_{4}\right]$ ) under the assumption that the main contribution is white noise. The energy density $u$, in units of 


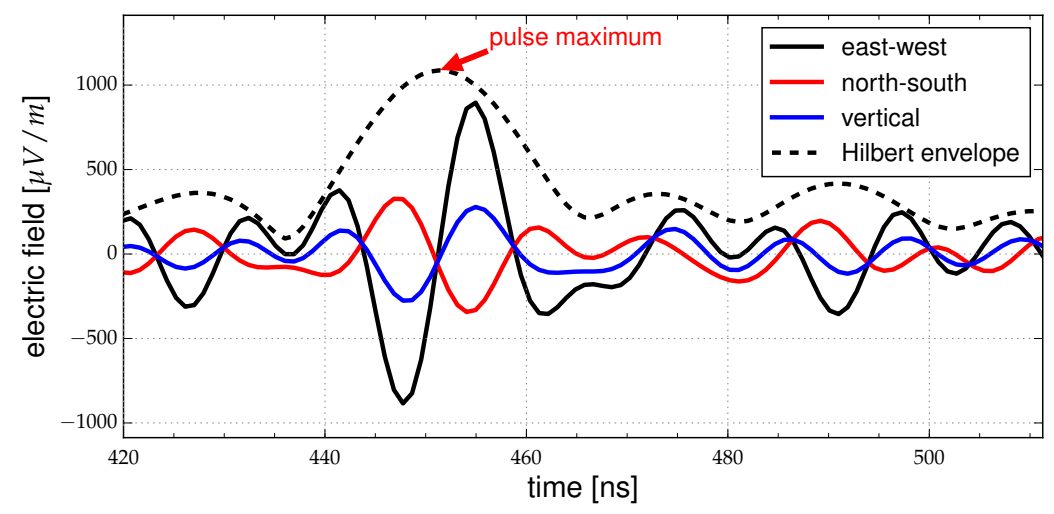

Figure 1: Reconstructed electric field trace of one of the measured cosmic ray radio events. An upsampling by a factor of five was applied. The shown Hilbert envelope (dashed line) is the square root of the quadratic sum of the Hilbert envelopes of the three polarization components.

$\mathrm{eV} / \mathrm{m}^{2}$, is given by

$$
u=\varepsilon_{0} c\left(\Delta t \sum_{t_{1}}^{t_{2}}\left|\vec{E}\left(t_{i}\right)\right|^{2}-\Delta t \frac{t_{2}-t_{1}}{t_{4}-t_{3}} \sum_{t_{3}}^{t_{4}}\left|\vec{E}\left(t_{i}\right)\right|^{2}\right),
$$

where $\varepsilon_{0}$ is the vacuum permittivity, $c$ is the speed of light in vacuum, $\Delta t$ is the size of one time bin. To approximate the uncertainty the noise level is used. In addition, the amplification of the signal chain adds $5 \%$ uncertainty in $u$ that is added in quadrature.

We apply quality cuts on the data of the surface detector [24]. The most important cuts are that the core position be surrounded by a hexagon of active stations and that the zenith angle of the incoming direction be less than $55^{\circ}$. Furthermore, we require that the reconstructed incoming direction from the radio and the surface detectors agree within $20^{\circ}$, that the event not be recorded during thunderstorms and we impose a cut on the measured polarization to efficiently reject noise pulses. 134 events remain after all cuts.

\section{Radiation energy and energy estimator}

To reconstruct the radiation energy, i.e., the energy that has been transferred from the cosmic ray into radiation in the 30 to $80 \mathrm{MHz}$ regime, the energy density - measured at the individual radio stations - is interpolated spatially and integrated. We use a two-dimensional lateral signal distribution function that describes all main features seen in simulated and measured cosmic ray radio events [17]. We parametrize the function with four free parameters, namely the amplitude $A$, the slope parameter $\sigma$ and the particle core position $\vec{r}_{\text {core }}$. The remaining constants $C_{0}-C_{4}$ are estimated from Monte Carlo simulations for the AERA site (see Tab. 1). Thus we find

$$
u(\vec{r})=A\left[\exp \left(\frac{-\left(\vec{r}+C_{1} \vec{e}_{\vec{v} \times \vec{B}}-\vec{r}_{\text {core }}\right)^{2}}{\sigma^{2}}\right)-C_{0} \exp \left(\frac{-\left(\vec{r}+C_{2} \vec{e}_{\vec{v} \times \vec{B}}-\vec{r}_{\text {core }}\right)^{2}}{\left(C_{3} e^{C_{4} \sigma}\right)^{2}}\right)\right] .
$$

All coordinates are in the shower plane and $\vec{r}$ denotes the station position. $\vec{e}_{\vec{v} \times \vec{B}}$ is the unit vector pointing into the polarization direction of the geomagnetic emission. The LDF is fitted to the data 

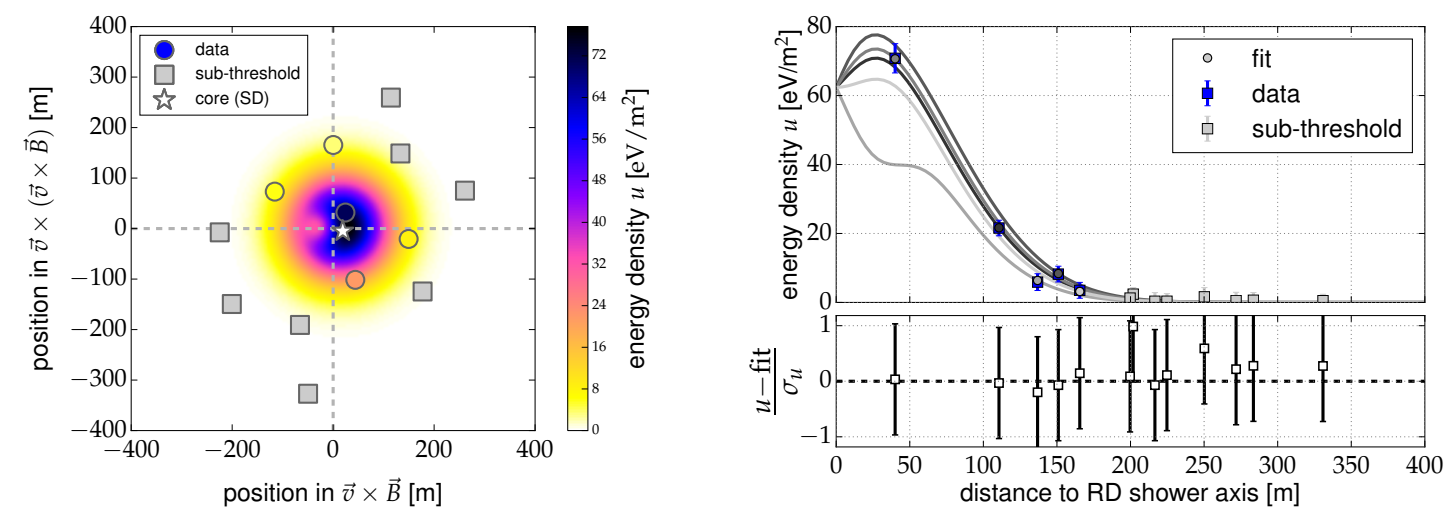

Figure 2: Lateral signal distribution of an air shower with an energy of $0.65 \mathrm{EeV}$ and arriving at a zenith angle of $21^{\circ}$ and from $31^{\circ}$ east of north. Left: Two dimensional distribution of the RD energy density in the shower plane. The coordinate origin is the RD shower core. The star indicates the SD core position. Circles and squares mark the positions of the radio stations. The color shows the measured energy density. Stations with signal below threshold are marked with gray squares. The background map shows the LDF parametrization. Right: One-dimensional projection of the LDF. Blue squares show the measured energy density and gray squares denote the sub-threshold stations. Gray circles denote the value of the LDF parametrization at the position of the measurement. The radial fall-off of the LDF along a line connecting the radio core position with each station position is shown as gray curves. Also shown are the residuals in units of the uncertainty of the measurement.

using a chi-square minimization. In case of low station multiplicity, the particle core position is taken from the SD reconstruction, which enables us to also use events with signals in only three or four radio stations. After a quality cut on the LDF fit, 126 events remain. An example of one air shower of our data set is shown in Fig. 2.

The radiation energy is the spatial integral of the LDF and will be given in units of $\mathrm{eV}$. To obtain a cosmic ray energy estimator we correct for the different emission strengths at different angles $\alpha$ between the shower axis and the magnetic field by dividing the radiation energy by $\sin ^{2} \alpha$ :

$$
S_{\text {radio }}=\frac{1}{\sin ^{2} \alpha} \int_{\mathbb{R}^{2}} u(\vec{r}) \mathrm{d}^{2} \vec{r}=\frac{A \pi}{\sin ^{2} \alpha}\left(\sigma^{2}-C_{0} C_{3}^{2} e^{2 C_{4} \sigma}\right),
$$

where $\mathbb{R}^{2}$ denotes the shower plane. The formula is valid for values of $\alpha>10^{\circ}$. For smaller values of $\alpha$ the geomagnetic emission is not the dominant contribution and the $\sin ^{2} \alpha$ correction becomes invalid. However, due to the reduced emission strength the number of detections for arrival directions within $10^{\circ}$ of the geomagnetic axis is suppressed. In our data set all events have arrival directions further away from the magnetic field axis.

The dominant event by event uncertainties of the energy estimator are the uncertainty of the LDF fit, the antenna response pattern (10\%) and the temperature dependence of the amplifiers (8\%) that is not yet corrected for. The average fit uncertainty of $S_{\text {radio }}$ is $46 \%$ and reduces to $24 \%$ in case of events with five or more stations with signal. The absolute scale uncertainty of the energy estimator is dominated by the absolute scale uncertainty of the antenna response pattern (25\%) [21] and the analog signal chain (12\%) and amounts to 28\%. All uncertainties are added in quadrature. Please note that as the energy estimator scales quadratically with the cosmic ray 


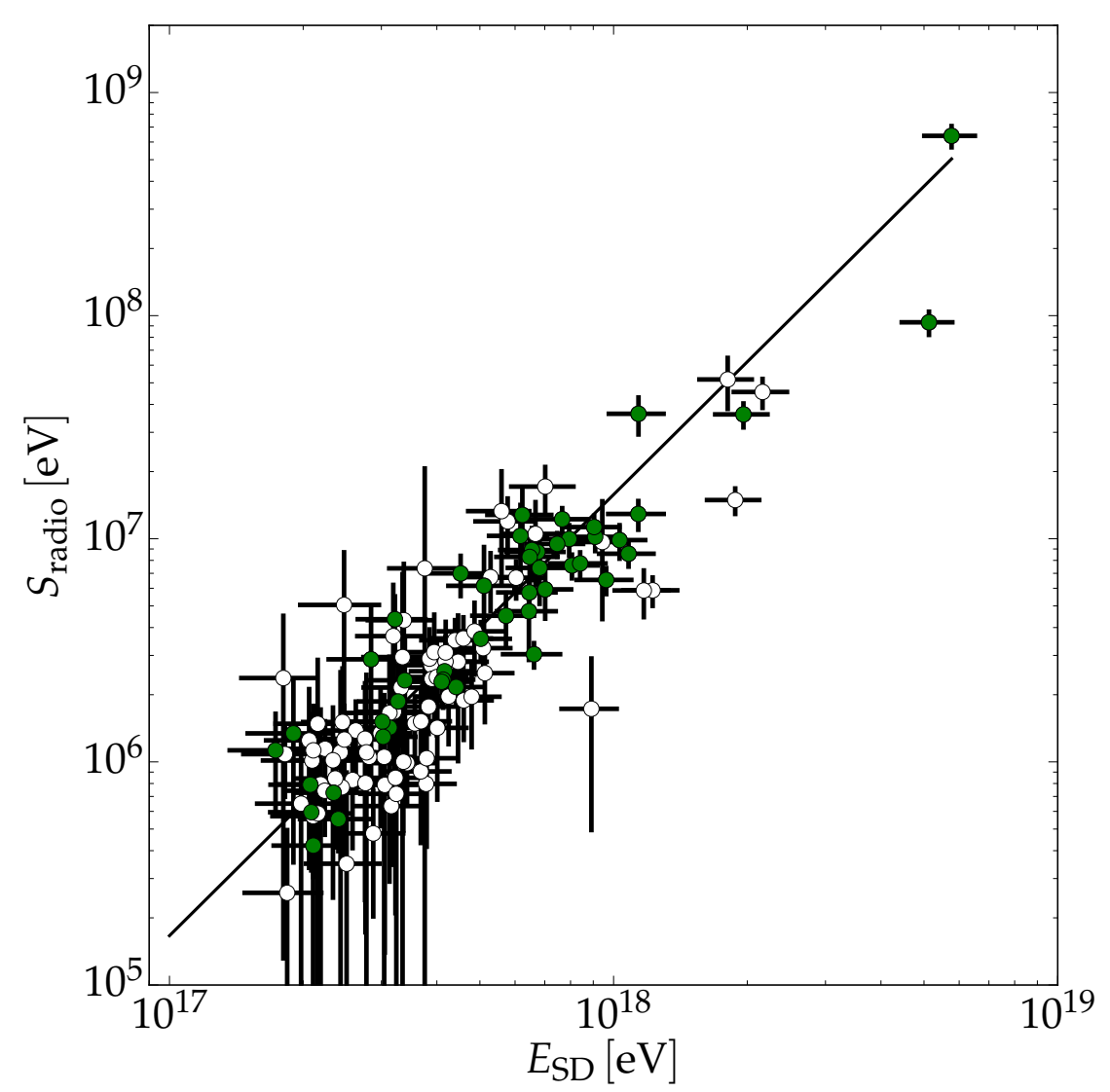

Figure 3: Correlation between the radio energy estimator $S_{\text {radio }}$ and the cosmic ray energy measured with the surface detector. Green filled circles denote air showers where the core position has been determined in the radio LDF fit, i.e., all air showers with at least five stations with signal. Open circles denote events with less than five stations with signal which use the SD core position. The black line is the best fit of the calibration function.

energy, the resulting uncertainty of the cosmic ray energy will be only half of the uncertainty of the energy estimator.

The energy estimator $S_{\text {radio }}$ is calibrated using air showers measured with AERA and the surface detector in coincidence. $S_{\text {radio }}$ shows a clear correlation with the cosmic ray energy measured by the surface detector (cf. Fig. 3). The calibration function $S_{\text {radio }}=A \times 10^{7} \mathrm{eV}\left(E_{\mathrm{SD}} / 10^{18} \mathrm{eV}\right)^{B}$ is obtained by maximizing a likelihood function that takes all measurement uncertainties, detector efficiencies and the steeply falling energy spectrum into account. This method was previously used in [25, 26] and is documented in [27]. The result of the calibration fit is $A=1.58 \pm 0.07$ and $B=1.98 \pm 0.04$. For a high quality subset of events with at least five radio stations with signal (47 events) the fit gives a compatible result of $A=1.60 \pm 0.08$ and $B=1.99 \pm 0.05$.

To estimate the energy resolution of AERA we subtract the known SD energy resolution from the scatter around the calibration curve, assuming that the energy estimators of the SD and RD are uncorrelated for a fixed cosmic ray energy. We find a resolution of $22 \%$ for the full data set and $17 \%$ for the high quality set. We also compare the observed and the expected scatter that can be calculated from the likelihood function and the uncertainties of the SD energy estimator and $S_{\text {radio }}$ 
discussed above and we find that the observed scatter is compatible with our expectation. As the radio energy estimator is calibrated with SD data, we inherit the systematic uncertainty of the SD energy scale. It is $14 \%$ at energies $\geq 10^{18} \mathrm{eV}$ [28] and increases to $16 \%$ at $10^{17.5} \mathrm{eV}$.

\section{The energy content of extensive air showers in the radio frequency range of $\mathbf{3 0}$ to $80 \mathrm{MHz}$}

We can generalize our results to other locations by normalizing the calibration function to the local magnetic field. Then, we can predict the radiation energy in the frequency range of 30 to $80 \mathrm{MHz}$ by

$$
E_{30-80 \mathrm{MHz}}=(15.8 \pm 0.7 \text { (stat) } \pm 6.7 \text { (sys) }) \mathrm{MeV}\left(\sin \alpha \frac{E}{10^{18} \mathrm{eV}} \frac{B_{\text {Earth }}}{0.24 \mathrm{G}}\right)^{2},
$$

where $E$ is the cosmic ray energy, $B_{\text {Earth }}$ denotes the local magnetic field strength and $0.24 \mathrm{G}$ is the magnetic field strength at the AERA site. We found that it is sufficient to correct only for the dominant geomagnetic emission process. However, this formula will become invalid for radio detectors at high altitudes because the amount of radiation energy decreases as - depending on the zenith angle - a significant part of the air shower is clipped away at the ground.

The direct measurement of the radiation energy holds further potential for a precise determination of the cosmic ray energy scale. Radio emission originates only from the electromagnetic part of an air shower. Therefore, the radiation energy can be predicted by Monte Carlo simulations from first principles [16, 29], as the emission arises from the acceleration/deceleration of charges which is described by classical electrodynamics [30]. In addition, no relevant propagation uncertainties arise as the atmosphere is transparent for radio emission. Then, in turn, the energy scale of the radio detector can be propagated to the other detection techniques.

\section{Conclusions}

We have presented a direct measurement of the radiation energy of an extensive air shower in the 30 to $80 \mathrm{MHz}$ regime. The AERA radio stations are well calibrated which enables the reconstruction of the energy density of the radio pulse at each detector position. The radiation energy is then the integral of the two-dimensional lateral signal distribution function that is fitted to the data. We measure $15.8 \mathrm{MeV}$ of radiation energy for a $1 \mathrm{EeV}$ cosmic ray arriving perpendicular to the local magnetic field.

As a cosmic ray energy estimator we use this radiation energy corrected for different emission strengths at different angles between the shower axis and the geomagnetic field. We calibrate the energy estimator using the reconstructed energy from the surface detector of the Pierre Auger Observatory and find that the radio energy estimator scales quadratically with the cosmic ray energy. We analyze the scatter around the calibration and find an energy resolution of the radio detector of $22 \%$. The resolution improves to $17 \%$ for a high quality subset of the data where only events with at least five radio stations with signal are used.

We generalize our result by normalizing the calibration function to the local magnetic field strength at the Auger site. This leads to a universal prediction of the radiation energy that can be used at any location. 
Table 1: Parameters $C_{0}-C_{4}$ of Eq. (3.1). $C_{3}=16.25 \mathrm{~m}$ and $C_{4}=0.0079 \mathrm{~m}^{-1}$. The zenith angle dependent values used to predict the emission pattern are given for zenith angle bins up to $60^{\circ}$.

\begin{tabular}{cccc}
\hline zenith angle & $C_{0}$ & $C_{1}[\mathrm{~m}]$ & $C_{2}[\mathrm{~m}]$ \\
\hline $0^{\circ}-10^{\circ}$ & 0.41 & $8.0 \pm 0.3$ & $-21.2 \pm 0.4$ \\
$10^{\circ}-20^{\circ}$ & 0.41 & $10.0 \pm 0.4$ & $-23.1 \pm 0.4$ \\
$20^{\circ}-30^{\circ}$ & 0.41 & $12.0 \pm 0.3$ & $-25.5 \pm 0.3$ \\
$30^{\circ}-40^{\circ}$ & 0.41 & $20.0 \pm 0.4$ & $-32.0 \pm 0.6$ \\
$40^{\circ}-50^{\circ}$ & 0.46 & $25.1 \pm 0.9$ & $-34.5 \pm 0.7$ \\
$50^{\circ}-60^{\circ}$ & 0.71 & $27.3 \pm 1.0$ & $-9.8 \pm 1.5$ \\
\hline
\end{tabular}

\section{References}

[1] LOPES Collaboration, H. Falcke et al., Detection and imaging of atmospheric radio flashes from cosmic ray air showers, Nature 435 (2005) 313-316.

[2] Codalema Collaboration, D. Ardouin et al., Radioelectric Field Features of Extensive Air Showers Observed with CODALEMA, Astropart. Phys. 26 (2006) 341-350.

[3] Pierre Auger Collaboration, S. Acounis, D. Charrier, T. Garçon, C. Rivière, and P. Stassi, Results of a self-triggered prototype system for radio-detection of extensive air showers at the Pierre Auger Observatory, J. Instrum. 7 (2012) P11023.

[4] C. Glaser for the Pierre Auger Collaboration, Energy estimation for cosmic rays measured with the Auger Engineering Radio Array, AIP Conf. Proc. 1535 (2013) 68-72.

[5] Tunka-Rex Collaboration, F. G. Schröder et al., The Tunka Radio Extension (Tunka-Rex): Status and First Results, Proc. 33rd ICRC, Rio de Janeiro, Brazil (2013) [1308 . 0910].

[6] LOPES Collaboration, W. Apel et al., Reconstruction of the energy and depth of maximum of cosmic-ray air showers from LOPES radio measurements, Phys. Rev. D 90 (2014) 062001, [1408.2346v1].

[7] T. Huege, The renaissance of radio detection of cosmic rays, Braz J Phys 44 (2014) 520-529.

[8] Codalema Collaboration, D. Ardouin et al., Geomagnetic origin of the radio emission from cosmic ray induced air showers observed by CODALEMA, Astropart. Phys. 31 (2009) 192-200.

[9] J. H. Hough and J. R. Prescott in Proceedings of the VI Interamerican Seminar on Cosmic Rays, vol. 2, p. 527, Universidad Mayor de San Andres, La Paz, Bolivia, 1970.

[10] J. R. Prescott, J. H. Hough, and J. K. Pidcock, Mechanism of radio emission from extensive air showers, Nature (London) Phys. Sci. 223 (1971) 109-110.

[11] V. Marin for the Codalema Collaboration, Charge excess signature in the Codalema data. interpretation with SELFAS2, Proc. 32nd ICRC, Beijing, China 1 (2011) 291.

[12] P. Schellart, S. Buitink, A. Corstanje, J. Enriquez, H. Falcke, J. Hörandel, M. Krause, A. Nelles, J. Rachen, O. Scholten, S. ter Veen, S. Thoudam, and T. Trinh, Polarized radio emission from extensive air showers measured with LOFAR, J. Cosmol. Astropart. P. 10 (2014) 014, [1406.1355].

[13] Pierre Auger Collaboration, A. Aab et al., Probing the radio emission from air showers with polarization measurements, Phys. Rev. D 89 (2014) 52002. 
[14] K. Werner and O. Scholten, Macroscopic treatment of radio emission from cosmic ray air showers based on shower simulations, Astropart. Phys. 29 (2008) 393-411.

[15] M. Ludwig and T. Huege, REAS3: Monte Carlo simulations of radio emission from cosmic ray air showers using an end-point formalism, Astropart. Phys. 34 (2011) 438-446.

[16] J. Alvarez-Muñiz, W. R. Carvalho, and E. Zas, Monte Carlo simulations of radio pulses in atmospheric showers using ZHAireS, Astropart. Phys. 35 (2012) 325-341, [11 07.1189 ].

[17] A. Nelles, S. Buitink, H. Falcke, J. R. Hörandel, T. Huege, and P. Schellart, A parameterization for the radio emission of air showers as predicted by CoREAS simulations and applied to LOFAR measurements, Astropart. Phys. 60 (2015) 13-24.

[18] A. Nelles et al., A lateral distribution function for the radio emission of air showers, Proc. 34th ICRC, The Hague, The Netherlands (2015).

[19] J. Schulz for the Pierre Auger Collaboration, Status and prospects of the Auger Engineering Radio Array, Proc. 34th ICRC, The Hague, The Netherlands (2015).

[20] Pierre Auger Collaboration, A. Aab et al., The Pierre Auger Cosmic Ray Observatory, accepted for publication in Nucl. Instrum. Meth. A (2015) [1502.01323].

[21] Pierre Auger Collaboration, P. Abreu et al., Antennas for the detection of radio emission pulses from cosmic-ray induced air showers at the Pierre Auger Observatory, J. Instrum. 7 (2012) P10011.

[22] S. Argirò, S. Barroso, J. Gonzalez, L. Nellen, T. Paul, T. Porter, L. Prado Jr., M. Roth, R. Ulrich, and D. Veberič, The Offline software framework of the Pierre Auger Observatory, Nucl. Instrum. Meth. A $\mathbf{5 8 0}$ (2007) 1485-1496.

[23] Pierre Auger Collaboration, P. Abreu et al., Advanced functionality for radio analysis in the Offline software framework of the Pierre Auger Observatory, Nucl. Instrum. Meth. A 635 (2011) 92-102.

[24] A. Schulz for the Pierre Auger Collaboration, The measurement of the energy spectrum of cosmic rays above $3 \times 10^{17} \mathrm{eV}$ with the Pierre Auger Observatory, Proc. 33rd ICRC, Rio de Janeiro, Brazil (2013) [1307.5059v1].

[25] I. Maris for the Pierre Auger Collaboration, Measurement of the Energy Spectrum of Cosmic Rays above $3 \times 10^{17} \mathrm{eV}$ at the Pierre Auger Observatory, Proceedings of Science EPS-HEP2013 (2013) 405.

[26] Pierre Auger Collaboration, A. Aab et al., Muons in air showers at the Pierre Auger Observatory: Mean number in highly inclined events, Phys. Rev. D 91 (2015) 032003.

[27] H. P. Dembinski, B. Kégl, I. C. Maris, M. Roth, and D. Veberič, A likelihood method to cross-calibrate air-shower detectors, submitted to Astropart. Phys. (2015) [1503.09027].

[28] V. Verzi for the Pierre Auger Collaboration, The energy scale of the Pierre Auger Observatory, Proc. 33rd ICRC, Rio de Janeiro, Brazil (2013).

[29] T. Huege, M. Ludwig, and C. W. James, Simulating radio emission from air showers with CoREAS, AIP Conf. Proc. 1535 (2013) 128-132.

[30] C. W. James, H. Falcke, T. Huege, and M. Ludwig, General description of electromagnetic radiation processes based on instantaneous charge acceleration in endpoints, Phys. Rev. E 84 (2011) 056602, [1007.4146]. 